\title{
Rituximab versus azathioprine as therapy for maintenance of remission for anti- neutrophil cytoplasm antibody-associated vasculitis (RITAZAREM): study protocol for a randomized controlled trial
}

\author{
Seerapani Gopaluni ${ }^{1 \dagger}$, Rona M. Smith ${ }^{1,4^{*}}$, Michelle Lewin ${ }^{1}$, Carol A. McAlear², Kim Mynard ${ }^{1}$, Rachel B. Jones ${ }^{1}$,
} Ulrich Specks ${ }^{3}$, Peter A. Merkel ${ }^{2}$, David R. W. Jayne ${ }^{1}$ and on behalf of the RITAZAREM Investigators

\begin{abstract}
Background: Rituximab is effective as therapy for induction of remission in anti-neutrophil cytoplasmic antibody (ANCA)-associated vasculitis (AAV). However, the effect of rituximab is not sustained, and subsequent relapse rates are high, especially in patients with a history of relapse. There is a need to identify whether maintenance therapy with rituximab is superior to the current standard of azathioprine or methotrexate for prevention of relapse following induction with rituximab.

Methods/design: RITAZAREM is an international, multicenter, open-label, randomized controlled trial designed to demonstrate the superiority of repeated doses of intravenous rituximab compared to daily orally administered azathioprine as a relapse prevention strategy in patients with AAV with relapsing disease who undergo induction of remission with rituximab. Patients with AAV will be recruited at the time of relapse and will receive rituximab and glucocorticoid induction therapy. If the disease is controlled by 4 months, patients will be randomized in a 1:1 ratio to receive rituximab (1000 mg every 4 months for five doses) or azathioprine ( $2 \mathrm{mg} / \mathrm{kg} /$ day) as maintenance therapy. Patients will be followed for a minimum of 36 months. The primary outcome is the time to disease relapse. It is estimated that 190 patients will need to be recruited to ensure that at least 160 are randomized.

Discussion: The RITAZAREM trial will provide the largest trial dataset for the use of rituximab as remission-induction therapy for patients with AAV comparing two remission-maintenance strategies following induction with rituximab, and explore whether prolonged B-cell depletion leads to sustained treatment-free remissions after discontinuation of immunosuppressive therapy.
\end{abstract}

Trial registration: ClinicalTrials.gov identifier: NCT01697267. Registered on 31 August 2012.

Keywords: ANCA-associated vasculitis, RITAZAREM, Rituximab, Azathioprine, Randomized clinical trial

\footnotetext{
* Correspondence: Ronasmith@doctors.net.uk

${ }^{\dagger}$ Equal contributors

'University of Cambridge Hospitals NHS Foundation Trust, Cambridge, UK

${ }^{4}$ Addenbrooke's Hospital, Box 118, Hills Road, Cambridge CB2 0QQ, UK

Full list of author information is available at the end of the article
} 


\section{Background}

Granulomatosis with polyangiitis (granulomatous with polyangiitis (GPA), Wegener's granulomatosis) and microscopic polyangiitis (MPA) are two major subgroups of anti-neutrophil cytoplasmic antibody (ANCA)-associated vasculitis (AAV). These conditions are characterized by leukocyte infiltration of blood vessel walls, fibrinoid necrosis, and vascular damage, and are usually associated with the presence of circulating ANCA. In Europe, AAV has an annual incidence of 19 per million and a prevalence of $100-250 /$ million [1].

Prior to the availability of effective treatment, AAV had a mortality of $93 \%$ within 2 years, primarily due to renal and respiratory failure [2]. The introduction of glucocorticoids (GC) and cyclophosphamide, now considered conventional immunosuppression for this disease, has markedly improved survival, inducing remission in approximately $80 \%$ of patients at 1 year. However, relapsing disease is common with over $50 \%$ of patients experiencing a relapse within 5 years [3-5].

Relapses of AAV lead to accrual of damage, both as a result of disease activity and increased exposure to immunosuppressive medications with their associated toxicities, especially GC, and at least $25 \%$ suffer severe early or late toxicity from these agents [6]. Strategies that prevent relapse will reduce consequent morbidity by reducing organ damage and cumulative drug toxicity. There is a need for safer therapies that lead to sustained, and ideally, drug-free remission in patients with relapsing disease in AAV.

B-lymphocytes have been implicated in the pathogenesis of AAV. Rituximab is a murine/human chimeric monoclonal antibody directed against the CD20 antigen found on the surface of B-lymphocytes, and results in Bcell depletion. Rituximab has been shown to be noninferior to cyclophosphamide as an induction agent, and it is now licensed for remission induction therapy in AAV $[7,8]$.

However, only $39 \%$ of the patients maintained relapsefree remission at 18 months following a single course of rituximab in the RAVE trial, indicating that induction with rituximab without maintenance therapy is unlikely to prevent future relapses in the majority of patients [9]. Retrospective analysis of fixed-interval, repeat-dose therapy with rituximab indicated efficacy of maintenance therapy with rituximab in this subgroup, but a persisting risk of relapse in longer-term follow-up after rituximab discontinuation [10-12].

\section{Rationale for the RITAZAREM trial}

The MAINRITSAN trial was designed to compare four cycles of rituximab $(500 \mathrm{mg} \times 2$ at 6 months then $500 \mathrm{mg}$ every 6 months $\times 3$ ) with maintenance therapy with azathioprine following induction with cyclophosphamide in patients with AAV, largely with patients with newly diagnosed disease [13]. Fixedinterval rituximab was shown to be more effective in maintaining remission at 28 months. The MAINRITSAN trial has provided valuable information on the use of rituximab as a maintenance agent in AAV, although several important questions remain unanswered. Firstly, the MAINRITSAN study was underpowered to assess the effect of repeat-dose rituximab in the subgroup with the most need of a remission-maintenance strategy, namely patients with relapsing disease (only 23 of 115 patients had relapsing disease). Secondly, in many countries rituximab has now been granted a license for remissioninduction in AAV, and is increasingly being used in place of cyclophosphamide. The optimal remission maintenance strategy following rituximab induction therapy is unknown. Thirdly, relapses do occur among patients treated with repeatedly-dosed rituximab, mostly in intervals of 6 more months [11, 12, 14]. Therefore, reducing the interval of repeated doses of rituximab to 4 months has the potential to further reduce relapse rates, and aims to maintain $100 \%$ B-cell depletion for the entire 24-month treatment phase. Finally, the total dose of rituximab for maintenance therapy in RITAZAREM is double that used in MAINRITSAN (5000 mg versus $2500 \mathrm{mg}$ ) which will permit comparison of the safety and longer-term efficacy of the two dosing regimens.

The RITAZAREM trial will address the question of the optimal treatment regimen for maintenance of remission following induction of remission with rituximab in AAV, and the possibility that prolonged B-cell depletion using high doses of rituximab, will lead, upon discontinuation of therapy, to sustained treatment-free remissions.

\section{Methods/design}

RITAZAREM is an international, multicenter, openlabel, randomized controlled trial designed to demonstrate the superiority of rituximab over azathioprine in the prevention of relapses in patients with AAV with relapsing disease (see Additional file 1 for the SPIRIT 2013 Checklist). This trial will also assess the risk of relapse after maintenance therapy is withdrawn at 24 months, the long-term safety of rituximab administration, and the optimal maintenance therapy in AAV following induction with rituximab.

The RITAZAREM trial has three phases:

1. An induction phase (months 0 to 4 ) in which eligible patients will be enrolled at the time of disease relapse and all will receive rituximab (4 weekly doses of $375 \mathrm{mg} / \mathrm{m}^{2}$ ) and GC 
2. A maintenance phase (months 4 to 24 ): 4 months after enrollment, participants who achieve remission (defined as a Birmingham Vasculitis Activity Score for Wegener's granulomatosis (BVAS/WG) $\leq 1$ [15] and prednisone/prednisolone dose $\leq 10 \mathrm{mg} /$ day) will be randomized in 1:1 ratio to receive $1000 \mathrm{mg}$ rituximab at 4-monthly fixed intervals or daily azathioprine maintenance therapy $(2 \mathrm{mg} / \mathrm{kg} /$ day $)$

3. A treatment-free follow-up period (minimum of 12 , maximum of 24 months)

\section{Randomization and allocation}

Enrollment and randomized allocation to treatment arm will be performed by an Internet-based website (TENELEA) provided by the Cambridge Clinical Trials Unit.

\section{Blinding}

This is an open-label study. Several previous vasculitis studies that have had major influences on practice and have stood up to re-examination have been unblinded, and similar endpoints have been used, with processes developed in data management to check and manage potential bias. Blinding would have necessitated the administration of sham infusions and placebo tablet medications to all participants, which would have increased the complexity and cost of the trial and exposed participants to unnecessary interventions. A pragmatic approach of having the primary endpoint, relapse, independently assessed by expert adjudicators blinded to treatment allocation was decided.

\section{Participants}

To be eligible, participants must be aged 15 years and above and should have a diagnosis of AAV according to Chapel Hill Consensus Conference definitions [16], along with a current or historical positive test for proteinase 3/myeloperoxidase anti-neutrophil cytoplasmic antibody (PR3/MPO ANCA) by enzyme-linked immunosorbent assay (ELISA). All patients must have disease relapse defined by one major or three minor disease activity items on the BVAS/WG and they must have previously achieved remission following at least 3 months of induction therapy, with a combination of GC and an immunosuppressive agent (cyclophosphamide, rituximab, methotrexate, or mycophenolate mofetil (MMF)).

Key exclusion criteria include the receipt of any biological B-cell-depleting agents within the previous 6 months, alemtuzumab, or anti-thymocyte globulin (ATG) within the last 12 months, or intravenously administered immunoglobulin (IVIg), plasma exchange, or anti-TNF treatment within the last 3 months. Patients with other multisystem autoimmune disease such as eosinophilic granulomatous with polyangiitis (eGPA), systemic lupus erythematosus (SLE), anti-glomerular basement membrane (GBM) disease or cryoglobulinaemic vasculitis, or history of malignancy within the past 5 years are also excluded. Additional file 2 lists the full inclusion and exclusion criteria for the study.

Participants will be recruited from many centers participating in the European Vasculitis Society (EUVAS), the Vasculitis Clinical Research Consortium (VCRC), and from centers in Japan, Australia, and New Zealand.

\section{Interventions}

See Additional file 3 for figure: RITAZAREM trial flow chart.

\section{Rituximab arm}

Induction phase: rituximab $375 \mathrm{mg} / \mathrm{m}^{2} /$ week $\times 4$ doses. This dose (as opposed to the 2-weekly 1000-mg induction dose, which has been shown to be equivalent to the fourdose schedule in retrospective series [17]), was selected because it is the licensed induction regimen for AAV.

Maintenance phase: $1000 \mathrm{mg}$ of rituximab repeated every 4 months for a total of five courses. Measureable B-cells usually return 4 months after a dose of rituximab, thus an every-4-month dosing interval aims to maintain 100\% B-cell depletion for the entire 24-month treatment phase. Additionally, in one series of every 6-month fixed dosing of rituximab $90 \%$ of relapses occurred in 4-6 months following a dose of rituximab; therefore, reducing the interval between doses of rituximab has the potential to further reduce relapse rates [14]. There is no clear evidence of dose-dependent toxicity for rituximab, and thus an every-4-month schedule was chosen to maximize efficacy.

\section{Control arm}

Induction phase: rituximab $375 \mathrm{mg} / \mathrm{m}^{2} /$ week $\times 4$ doses.

Maintenance phase: orally administered azathioprine with a target dose of $2 \mathrm{mg} / \mathrm{kg} /$ day (maximum $200 \mathrm{mg} /$ day) for 24 months. After 24 months, the dose will be reduced by $50 \%$ and it will be completely withdrawn at month 27 .

Patients who are intolerant to azathioprine will receive either methotrexate (administered orally or subcutaneously) at a maximum dose of $25 \mathrm{mg} /$ week (if estimated glomerular filtration rate is $>50 \mathrm{ml} / \mathrm{min}$ ), or $\mathrm{MMF}$ at a maximum dose of $2 \mathrm{~g} /$ day (if estimated glomerular filtration rate (GFR) is $\leq 50 \mathrm{ml} / \mathrm{min}$ ).

\section{Concomitant therapy \\ Glucocorticoids}

Induction: since evidence for optimal GC induction dosing in relapsing AAV is lacking, it was decided, to facilitate recruitment, to allow investigators to choose from one of two $\mathrm{GC}$ regimens to take into consideration variability in disease severity and local GC prescribing practices (Table 1). Patients may receive a maximum 
Table 1 Orally administered glucocorticoid (prednisone/ prednisolone) dosing in the RITAZAREM trial

\begin{tabular}{lllll}
\hline & \multicolumn{2}{l}{ Induction schedule A } & \multicolumn{2}{l}{ Induction schedule B } \\
(1 mg/kg group) & $2.5 \mathrm{mg} / \mathrm{kg}$ group) \\
\hline Week & $<60 \mathrm{~kg}$ & $\geq 60 \mathrm{~kg}$ & $<60 \mathrm{~kg}$ & $\geq 60 \mathrm{~kg}$ \\
0 & $50 \mathrm{mg} /$ day & $60 \mathrm{mg} /$ day & $25 \mathrm{mg} /$ day & $30 \mathrm{mg} /$ day \\
2 & $35 \mathrm{mg} /$ day & $45 \mathrm{mg} /$ day & $20 \mathrm{mg} /$ day & $25 \mathrm{mg} /$ day \\
4 & $25 \mathrm{mg} /$ day & $35 \mathrm{mg} /$ day & $17.5 \mathrm{mg} /$ day & $20 \mathrm{mg} /$ day \\
6 & $20 \mathrm{mg} /$ day & $25 \mathrm{mg} /$ day & $15 \mathrm{mg} /$ day & $17.5 \mathrm{mg} /$ day \\
8 & $15 \mathrm{mg} /$ day & $17.5 \mathrm{mg} /$ day & $12.5 \mathrm{mg} /$ day & $15 \mathrm{mg} /$ day \\
10 & $12.5 \mathrm{mg} /$ day & & $12.5 \mathrm{mg} /$ day & \\
12 & $10 \mathrm{mg} /$ day & & $10 \mathrm{mg} /$ day & \\
\hline
\end{tabular}

cumulative dose of $3000 \mathrm{mg}$ intravenously administered (IV) methylprednisolone, between 14 days prior to enrollment and 7 days after enrollment.

Maintenance: an orally administered GC (prednisone/ prednisolone or the equivalent) dose of $10 \mathrm{mg} /$ day or less is a requirement for randomization at 4 months. The GC dose is reduced to $5 \mathrm{mg} /$ day by month 6 , and is continued at $5 \mathrm{mg} /$ day until month 16 . The GC dose is then reduced to $2.5 \mathrm{mg} /$ day and completely withdrawn at month 20 .

\section{Other treatments}

\section{Prophylactic therapies}

Prophylaxis to prevent Pneumocystis (carinii) jiroveci infection and/or to prevent osteoporosis are strongly recommended but implementation is left to local practice.

\section{Plasma exchange}

Plasma exchange can be administered during the induction period following local practice at the discretion of the investigator. Rituximab will not be administered within the $48 \mathrm{~h}$ prior to receiving a plasma exchange treatment.

\section{Treatment of relapse}

Time to relapse is the primary endpoint of the study. $\mathrm{Pa}-$ tients experiencing their first minor relapse after randomization and before month 24 will remain on their randomized treatment and the dose of orally administered prednisone/prednisolone will be increased to $20 \mathrm{mg} /$ day for 1 week decreasing in daily $2.5-\mathrm{mg}$ increments each week until the dose is back to the dose before the relapse at which point the patient returns to the standard dosing schedule. The second minor relapse, or first major relapse, occurring before month 24, will result in the patient being withdrawn from protocolized treatment and the patient will be treated according to the investigator's discretion. Any relapse occurring after the 24-month treatment phase will be treated according to local best medical practice.

\section{Assessments}

Evaluations (including clinical, biochemical, and patientreported outcomes) will be performed at months $0,1.5$, $3,4,8,12,16,20,24,27,30,36$, and every 6 months until the last patient has completed 36 months in the study (see Additional file 4 - SPIRIT figure: RITAZAREM schedule of events). The maximum duration in the study for any individual is 48 months. Assessments will also be performed at the time of relapse. Data will be collected on paper Case Report Forms, and entered into an electronic database hosted by the Cambridge Clinical Trials Unit.

\section{Outcomes}

\section{Primary outcome}

1. Time to disease relapse (either minor or major relapse), reported at 24 months

\section{Secondary outcomes}

1. Proportion of patients who maintain remission at 24 and 48 months

2. Time to a major or second minor relapse

3. Cumulative accrual of damage as measured by the Combined Damage Assessment (CDA) score [18]

4. Health-related quality of life as measured using 36-item Short Form Health Survey (SF-36), the European Quality of Life-5 Dimensions (EQ5D), and Patient Reported Outcomes Measurement Information System (PROMIS) short forms for fatigue, pain, physical function, and patient global assessment [19]

5. Cumulative GC exposure

6. Severe adverse event rates and infection (treated with either intravenously or orally administered antibiotics) rates

\section{Power calculation}

Enrollment will be ongoing until 160 patients are randomized at their 4-month visits. It is anticipated that 190 patients will be required in order to randomize 160 patients. A power of $90 \%$ will be achieved under the alternative hypothesis of a hazard ratio of 0.42 at the $5 \%$ significance level with 58 observed relapses. Randomizing 160 patients will achieve this over the course of the study assuming a dropout rate up to $5 \%$ at 2 years and a relapse-free rate of $75 \%$ and $50 \%$ at 4 years in the experimental and control arms, respectively. The hazard ratio of 0.42 is based on assuming a relapse-free rate of $75 \%$ at 4 years in the rituximab arm versus $50 \%$ at 4 years in the standard treatment arm. 


\section{Analyses}

A statistical analysis plan, separate to the study protocol, will be finalized prior to any analyses being performed. The primary intention-to-treat analysis will be based on a Cox proportional hazard model. There will be a closed testing procedure. Hazard ratios and 95\% confidence intervals will be reported. First, the null hypothesis will be for a hazard ratio of 1 at all time points. If this is rejected at a $5 \%$ level then two further subhypotheses will be examined using time-varying covariates:

1. A hazard ratio of 1 up to 24 months post randomization (i.e. during treatment)

2. A hazard ratio of 1 after 24 months post randomization (i.e. post treatment)

\section{Funding, sponsor, and committee oversight}

RITAZAREM is funded by grants from Arthritis Research UK and Roche/Genentech. The Vasculitis Clinical Research Consortium (VCRC) (U54 AR057319 and U01 AR5187404) is part of the Rare Diseases Clinical Research Network (RDCRN), an initiative of the Office of Rare Diseases Research (ORDR), National Center for Advancing Translational Science (NCATS). The VCRC is funded through collaboration between NCATS, and the National Institute of Arthritis and Musculoskeletal and Skin Diseases, and has received funding from the National Center for Research Resources (U54 RR019497). The primary sponsor is Cambridge University Hospitals NHS Foundation Trust and there are collaboration and data-sharing agreements with the University of Pennsylvania and the University of Miyazaki Hospital and Okayama University Graduate School of Medicine, Dentistry, and Pharmaceutical Sciences in Japan. A NIAMS Data and Safety Monitoring Board (DSMB) will provide oversight of this study. This is a standing DSMB that provides such oversight for all VCRC research studies, including randomized trials. The DSMB will meet at least every 6 months to discuss the study progress and more frequently, if needed. The Trial Steering Committee will provide overall supervision of the study on behalf of the principal funder, Arthritis Research UK. The Trial Steering Committee will monitor the progress of the study and maximize the chances of completing the study within the agreed time scale and budget. The Trial Management Committee is responsible for the design, conduct and overall management of the study. Cambridge Clinical Trials Unit has provided support in all phases of trial design, conduct, and management for this trial.

\section{Patient participation}

The design of the study was presented at multiple local, national, and international meetings with patient attendees with positive review and input received from patients with AAV. The Patient Information Sheet was reviewed and commented upon by a patient panel prior to submission to the Ethics Committee.

\section{Discussion}

It is becoming increasingly clear that patients with AAV with relapsing disease need different treatment strategies compared to patients with nonrelapsing disease. RITAZAREM is the largest clinical trial recruiting only the subgroup of patients with relapsing AAV.

The RITAZAREM trial will add to our knowledge on the use of rituximab both as an induction and a maintenance therapy in this particular patient population. This trial will also be the largest study to examine the outcome of induction with rituximab at 4 months in patients with relapsing AAV.

The MAINRITSAN study has shown that fixedinterval re-dosing of rituximab is effective in patients with AAV with new or relapsing disease but the RITAZAREM trial will also answer the following specific questions:

- Is fixed-interval rituximab re-dosing therapy superior to azathioprine in maintaining remission following rituximab induction in relapsing patients?

- Does a higher rituximab dose confer long-term benefit after discontinuation of therapy, without significant drug-related toxicity?

Data generated from the RITAZAREM study will compliment the already-available results of the MAINRITSAN trial [13] and published cohort studies [11, 12, 14]. RITAZAREM is an example of an academic-led clinical trial in a rare disease, facilitated by highly collaborative work of established regional vasculitis networks in Europe, North America, Japan, and Australia/New Zealand. It also demonstrates collaboration between academia and the pharmaceutical industry and continues the repurposing of a drug originally developed for lymphoma but subsequently licensed for the autoimmune disease rheumatoid arthritis.

\section{Trial status}

Enrollment was completed on 21 November 2016, and thus randomization will be complete by the end of March 2017. The primary endpoint will be reported in 2019, and the final trial report in 2020 .

\section{Additional files}

Additional file 1: SPIRIT_Fillable-checklist-RITAZAREM; populated SPIRIT Checklist. (DOC 122 kb) 
Additional file 2: Inclusion and exclusion criteria for RITAZAREM trial; full list of enrollment requirements. (DOC $34 \mathrm{~kb}$ )

Additional file 3: RITAZAREM trial flow chart; RITAZAREM study schema. (DOC $68 \mathrm{~kb}$ )

Additional file 4: SPIRIT figure: RITAZAREM schedule of events; populated SPIRIT figure. (DOC $75 \mathrm{~kb}$ )

\section{Abbreviations}

AAV: ANCA-associated vasculitis; ANCA: Anti-neutrophil cytoplasmic antibody; BVAS: Birmingham Vasculitis Activity Score; CDA: Combined Damage Assessment; DSMB: Data Safety Monitoring Board; eGPA: Eosinophilic granulomatous with polyangiitis; ELISA: Enzyme-linked immunosorbent assay; EQ5D: European Quality of Life-5 Dimensions; EUVAS: European Vasculitis Society; GBM: Glomerular basement membrane; GC: Glucocorticoids; GFR: Glomerular filtration rate; GPA: Granulomatous with polyangiitis; IV: Intravenous; IVlg: Intravenous immunoglobulin; MMF: Mycophenolate mofetil; MPA: Microscopic polyangiitis; MPO: Myeloperoxidase; NIAMS: The National Institute for Arthritis and Musculoskeletal and Skin Diseases: PR3: Proteinase 3; PROMIS: Patient Reported Outcomes Measurement Information System; SF36: Short Form 36; SLE: Systemic lupus erythematosus; VCRC: Vasculitis Clinical Research Consortium

\section{Acknowledgements}

This research was supported by the National Institute for Health Research (NIHR) Cambridge Biomedical Research Centre and the Cambridge Clinical Trials Unit (CCTU). Roche/Genentech have provided rituximab and financial support for the conduct of the study.

RITAZAREM is a joint venture of the European Vasculitis Study Group (EUVAS) and the Vasculitis Clinical Research Consortium (VCRC).

\section{Funding}

Arthritis Research UK: The National Institute for Arthritis and Musculoskeletal and Skin Diseases (NIAMS); The National Center for Advancing Translational Science (NCATS); Roche/Genentech; Japanese Ministry of Health, Labour, and Welfare.

\section{Availability of data and materials}

Data sharing not applicable to this article as no datasets were generated or analyzed during the current study.

\section{Authors' contributions}

RMS, ML, CAM, RBJ, US, PAM, and DRWJ have been involved in the conception and design of the study. RMS, ML, KM, CAM, PAM, and DRWJ have been involved obtaining ethical and regulatory approvals. SG, RMS, and $M L$ have drafted the manuscript. KM, RBJ, US, PAM, and DRWJ have critically reviewed the manuscript for important intellectual content. All authors have approved the final version of the paper for publication.

\section{Competing interests}

SG: none. RMS; Research support from Roche, Genzyme/Sanofi, and AstraZenica/Medimmune. Lecture fees from Roche. ML: none. CAM: none. KM: none. RBJ: consulting for ChemoCentryx. Academic secondment with GlaxoSmithKline 2011-2013. US: consulting for Genentech/Roche. PAM: Consulting for Actelion, Alexion, Bristol Myers Squibb, ChemoCentryx, Genzyme/Sanofi, GlaxoSmithKline, Genentech/Roche, PrincipioBio. Research support from Actelion, Bristol Myers Squibb, Celgene, ChemoCentryx, Genentech/Roche, GlaxoSmithKline. DRWJ: consulting for Alexion, ChemoCentryx, Genzyme/Sanofi, GlaxoSmithKline, Genentech/Roche, and Takeda. Research support from ChemoCentryx, Genentech/Roche, Genzyme/ Sanofi, Medimmune, and GlaxoSmithKline.

\section{Consent for publication}

Not applicable.

\section{Ethics approval and consent to participate}

An initial favourable ethical opinion was granted by NRES Committee East of England - Cambridge South: REC reference: 12/EE/0230 on 24 July 2012. Informed consent has been obtained from all participants in this study.

\section{Author details}

'University of Cambridge Hospitals NHS Foundation Trust, Cambridge, UK. ${ }^{2}$ Division of Rheumatology and Department of Epidemiology and Biostatistics, University of Pennsylvania, Philadelphia, PA, USA. ${ }^{3}$ Department of Pulmonary and Critical Care Medicine, Mayo Clinic, Rochester, MN, USA.

${ }^{4}$ Addenbrooke's Hospital, Box 118, Hills Road, Cambridge CB2 OQQ, UK.

Received: 16 November 2016 Accepted: 20 February 2017

Published online: 07 March 2017

\section{References}

1. Watts RA, Gonzalez-Gay MA, Lane SE, Garcia-Porrua C, Bentham G, Scott DG. Geoepidemiology of systemic vasculitis: comparison of the incidence in two regions of Europe. Ann Rheum Dis. 2001;60(2):170-2.

2. Frohnert PP, Sheps SG. Long-term follow-up study of periarteritis nodosa. Am J Med. 1967;43(1):8-14.

3. de Groot K, Harper L, Jayne DR, Flores Suarez LF, Gregorini G, Gross WL, et al. Pulse versus daily oral cyclophosphamide for induction of remission in antineutrophil cytoplasmic antibody-associated vasculitis: a randomized trial. Ann Intern Med. 2009:150(10):670-80

4. Wegener's Granulomatosis Etanercept Trial Research Group. Etanercept plus standard therapy for Wegener's granulomatosis. N Engl J Med. 2005;352(4):351-61

5. Jayne D, Rasmussen N, Andrassy K, Bacon P, Tervaert JW, Dadoniene J, et al. A randomized trial of maintenance therapy for vasculitis associated with antineutrophil cytoplasmic autoantibodies. N Engl J Med. 2003:349(1):36-44.

6. Seo P, Min YI, Holbrook JT, Hoffman GS, Merkel PA, Spiera R, et al. Damage caused by Wegener's granulomatosis and its treatment: prospective data from the Wegener's Granulomatosis Etanercept Trial (WGET). Arthritis Rheum. 2005;52(7):2168-78.

7. Jones RB, Tervaert JW, Hauser T, Luqmani R, Morgan MD, Peh CA, et al. Rituximab versus cyclophosphamide in ANCA-associated renal vasculitis. N Engl J Med. 2010:363(3):211-20.

8. Stone JH, Merkel PA, Spiera R, Seo P, Langford CA, Hoffman GS, et al. Rituximab versus cyclophosphamide for ANCA-associated vasculitis. N Engl J Med. 2010;363(3):221-32

9. Specks U, Merkel PA, Seo P, Spiera R, Langford CA, Hoffman GS, et al. Efficacy of remission-induction regimens for ANCA-associated vasculitis. N Engl J Med. 2013;369(5):417-27.

10. Alberici F, Smith RM, Jones RB, Roberts DM, Willcocks LC, Chaudhry A, et al. Long-term follow-up of patients who received repeat-dose rituximab as maintenance therapy for ANCA-associated vasculitis. Rheumatology (Oxford). 2015;54(7):1153-60

11. Cartin-Ceba R, Golbin JM, Keogh KA, Peikert T, Sanchez-Menendez M, Ytterberg SR, et al. Rituximab for remission induction and maintenance in refractory granulomatosis with polyangiitis (Wegener's): ten-year experience at a single center. Arthritis Rheum. 2012;64(11):3770-8.

12. Pendergraft 3rd WF, Cortazar FB, Wenger J, Murphy AP, Rhee EP, Laliberte $K A$, et al. Long-term maintenance therapy using rituximab-induced continuous B-cell depletion in patients with ANCA vasculitis. Clin J Am Soc Nephrol. 2014;9(4):736-44.

13. Guillevin L, Pagnoux C, Karras A, Khouatra C, Aumaitre O, Cohen P, et al. Rituximab versus azathioprine for maintenance in ANCA-associated vasculitis. N Engl J Med. 2014;371(19):1771-80.

14. Smith RM, Jones RB, Guerry MJ, Laurino S, Catapano F, Chaudhry A, et al. Rituximab for remission maintenance in relapsing antineutrophil cytoplasmic antibody-associated vasculitis. Arthritis Rheum. 2012:64(11):3760-9.

15. Stone JH, Hoffman GS, Merkel PA, Min YI, Uhlfelder ML, Hellmann DB, et al. A disease-specific activity index for Wegener's granulomatosis: modification of the Birmingham Vasculitis Activity Score. International Network for the Study of the Systemic Vasculitides (INSSYS). Arthritis Rheum. 2001:44(4):912-20.

16. Jennette JC, Falk RJ, Bacon PA, Basu N, Cid MC, Ferrario F, et al. 2012 revised International Chapel Hill Consensus Conference Nomenclature of Vasculitides. Arthritis Rheum. 2013:65(1):1-11.

17. Jones RB, Ferraro AJ, Chaudhry AN, Brogan P, Salama AD, Smith KG, et al. A multicenter survey of rituximab therapy for refractory antineutrophil cytoplasmic antibody-associated vasculitis. Arthritis Rheum. 2009;60(7):2156-68.

18. Seo P, Luqmani RA, Flossmann O, Hellmich B, Herlyn K, Hoffman GS, et al. The future of damage assessment in vasculitis. J Rheumatol. 2007;34(6):1357-71.

19. Hanmer J, Feeny D, Fischhoff B, Hays RD, Hess R, Pilkonis PA, et al. The PROMIS of QALYS. Health Qual Life Outcomes. 2015;13:122. 